\title{
Interactive Design of Picture Album Based on Painting Scheme and Texture Experience
}

\author{
Kai Yao ${ }^{1, \text { a }}$, Jie Tian ${ }^{2, b^{*}}$ \\ ${ }^{l}$ Fine art of Fujian Normal University, Minhou, Fuzhou, Fujian, China \\ ${ }^{2}$ Straits Institute of Minjiang University, Minhou, Fuzhou, Fujian, China \\ 314510671@qq.com \\ b*308986977@qq.com
}

\begin{abstract}
The schema language and extremely rich texture effect of lacquer painting make it much more different than other kinds of painting. This paper discusses the interactive design of picture albums from the perspective of schema language and texture language of lacquer painting. It elaborates the mechanism of making users to experience both schema and texture language of the functional modules and interface design. On one hand, it is expected that this experience, which is different from other kinds of painting, will enable the viewers to understand deeply and learn more about lacquer painting, especially their uniqueness, extensibility, fun and re-creation. On the other hand, it is also expected to provide some thinking for the digital development of lacquer painting creation in the future.
\end{abstract}

Keywords: Lacquer painting schema, Lacquer painting skin texture, Picture album, interaction

\section{基于漆画图式与肌理体验的画册交互式设计}

\author{
姚凯 ${ }^{1, \mathrm{a}}$, 田洁 ${ }^{2, \mathrm{~b} *}$
}

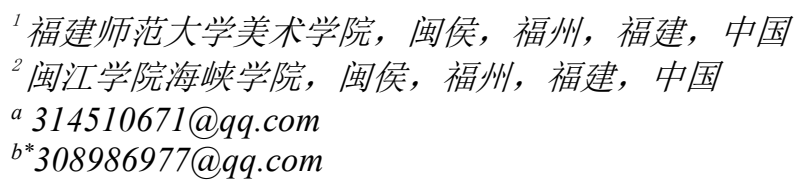

\section{摘要}

漆画的图式语言以及极其丰富的肌理效果使其跟其他画种有明显的不同，本文从漆画的图式语言和肌 理语言两个角度谈画册的交互式设计，阐述在画册的交互式设计中，如何在功能模块、界面设计上让 用户体验图式语言和肌理语言，以期这种不同于其他画种的体验能够让观者更深层次的了解和学习漆 画画种, 了解其图式语言和肌理语言的独特性、可延展性、趣味性和再创作性; 另外也可给漆画创作 未来的数字化的发展提供一定的思考。

关键词: 漆画图式，漆画肌理，画册，交互

\section{1. 前言}

我国漆艺的历史源远流长, 浙江萧山跨湖桥遗址出 土了距今八千多年的漆弓, 是我国目前发现的最早的漆 文物。在两千多年前的战国时期出土的运用较为写实手 法绘制的在山水间起舞的女子的漆奁, 这是最早的漆画 雏形, 可以说, 中国现代的漆画是从古代传统漆器中催 生出来的。上世纪三十年代左右, 中国开启了现代漆画 的探索时期。六十年代时候越南漆画来到国内展览, 给
与了国内漆画工作者们全新的视觉体验。八十年代第六 届全国美展开始, 漆画独立进行展览, 漆画参展获奖人 数不断增加。【11这个世纪初, 中国美术家协会漆画艺 术委员会成立, 艺委会通过举办各种规格和层级的展 览、组织培养一大批中青年漆画创作队伍等手段，极大 的提升了漆画队伍整体创作水平。通过近几十年的发 展，现代漆画没有拘泥于平光亮传统工艺的束缚，并注 入了漆画工作者们的主观创作思维和个人情感。这些漆 画作品形式各异、风格多样，同时部分画作体现了与其 他画种结合的创作手法, 可以说呈现出蓬勃发展的景 
象。

\section{2. 漆画的图式和肌理}

\section{1 漆画的图式}

所谓图式, 是人脑中已有的知识经验的网络。“图 式” 这个概念最早是由康德提出, 认为图式是 “潜藏在 人类心灵深处的”一种技术、一种技巧。“2绘画图式 是一种传达情感和经验的视觉符号样式。漆画的图式特 征非漆艺也非其他任何画种的图式特征, 漆画有其自身 独特的纯粹的漆画语境, 漆画的图式语言非国画图式的 运用泼、酒、传、擦的 “气韵传神” , 非油画图式的油 彩的揉、挫、扫、拉的笔触, 也非版画的木刻、光刻、 蚀刻的方式。大漆可以起皱、变涂、磨显、罩染、与其 他材料如蛋壳、瓦灰、金银粉等结合形成材料化的图式 语言。例如大漆勾画起皱的线、蛋壳镶嵌的线, 这些属 于起高的凸线。或通过线的图案留空, 将线周围的肌理 进行堆塑, 即可留出凹线, 同样, 通过疏密交织的刻线 再填彩也可做出凹线。这种以线为基底和骨骼, 通过线 性间隔将不同质料融汇表现、线性的图式与丰富的肌理 语言关系共生了漆画画境。

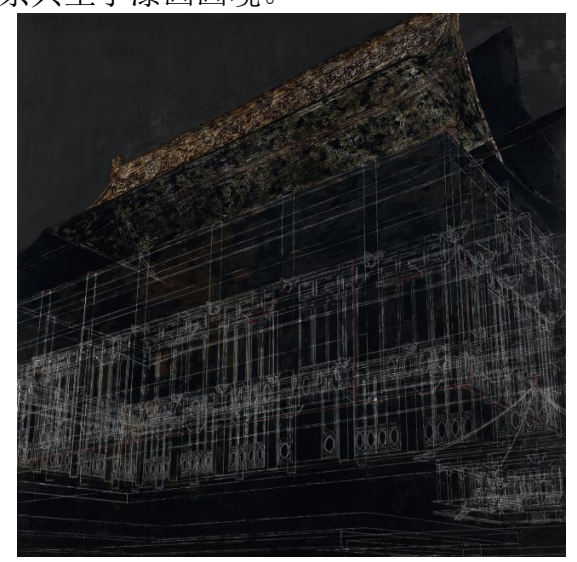

图 1 图式语言 (马蕾《如跂斯翼・如矢斯棘》)

\section{2 渎画的肌理}

首先是基于材料的漆画肌理。肌理是固态物质表面 的自身起伏和纹理结构, 是媒材的一种存在和表现形 式, 基本可以概括分为视觉层面上的肌理感受和触觉层 面上的肌理触感。传统的漆画追求平光亮的视觉效果, 视觉肌理打磨平整后呈现在平光亮表层效果之下。而今 漆画的衡量标准更加多元化, 越来越多的漆画创作者也 开始注重触觉肌理的研究。漆画的最基本的材料是天然 大漆。大漆独特的魅力便是其可以转印非常丰富的肌理 效果, 大漆本身在湿度高的情况下会快速结膜起皱, 这 跟当时的温度、湿度、漆调制的粘稠度都有关系, 会氧 化变色、干燥变化, 具有偶然性和不可控性; 同样, 其 他很多材料与大漆结合呈现出丰富的肌理效果, 比如运
用薄荷油、煤油、汽油等稀释剂调稀大漆使其具有更强 的流动性, 通过泼漆呈现出不同颜色、不同形态的大漆 相互交融渗透的肌理, 其意蕴或气势磅礴或轻松自在; 运用蛋壳材料通过破碎的蛋壳与底㴚镶嵌形成不规则 的冰裂纹; 运用色漆、瓦灰、生漆摚拌混合在画板上堆 髹, 干固后又会呈现出偏亚光的颗粒肌理; 用螺钿镶嵌 形成的细淢的光泽质感; 金银箔、金银粉、铝䇴粉会形 成金属散发的光泽, 其与大漆的温婉含蓄结合产生独特 的视觉肌理; 塑料薄膜、豆类、树叶、布匹、纸团、铁 丝、面粉等材料都可以与大漆结合创造出漆画创作者所 需要的肌理效果。可以说, 肌理的展现是漆画创作者常 用的情感表达的重要手段。

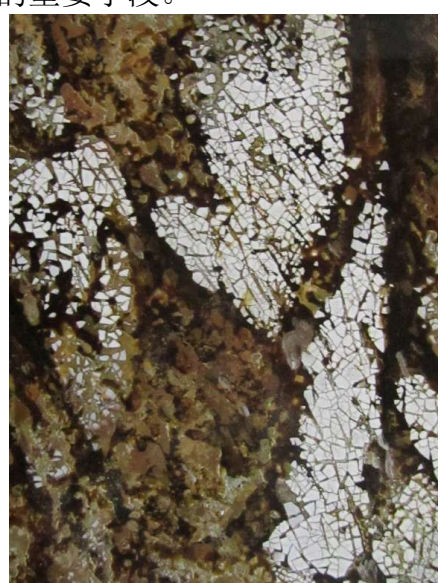

图 2 蛋壳肌理

其次是基于技法的漆画肌理。漆画的技法种类很 多, 一部分技法是在多年的创作中积累下来的经验之 谈, 另外一部分是漆画创作者在作画过程中偶然创作出 来的。漆画与其他画种不同的是漆画的各种技法会带来 较强的偶然性, 这种偶然性会带来意想不到的肌理效 果。主要的工艺技法有描绘、变涂（工具、材料起纹, 泼酒等)、镶嵌、堆髹等。

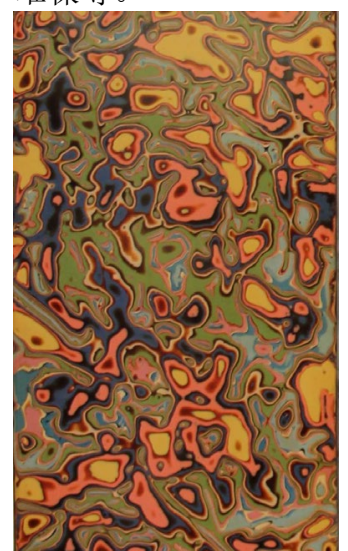

图 3 不同颜色大漆叠层打磨肌理

描绘就是描画的意思。一般勾勒、描线、描画这些 工艺手法都属于描绘的范畴。在《髹饰录》书中提到了 “描漆”、“描金”等描绘的手法; 泼酒的技法便是将 作画的材料根据自己的创作需求泼酒在画面上, 形成不 
同构成关系的具有较强偶然性的肌理, 如漆画大师李芝 卿创作的 “漂漆流彩” 的泼洒技法。镶嵌技法形式有三 种一平嵌、挖嵌和浮嵌, 平嵌一般螺钿镶嵌的方式采用 的比较多, 浮嵌多采用木、石等坚硬材质的镶嵌; 堆髹 的技法就是将大漆、漆粉或者瓦灰等材料根据创作意图 在画面中堆积, 这种技法自古旧有, 可以堆出浮雕的效 果, 从而进行鬆饰。

\section{3. 交互式画册设计创意}

交互界面是人机互动的桥梁, 良好的交互界面设计 可以有效的提升用户体验。现代科学的进步, 客观存在 的环境也可以模拟成人为创造的虚拟环境。比如 $A R 、 V R$ 技术可以给与虚拟的世界以强烈的沉浸感和代入感。传 统的绘画作品可以通过技术变成再创造的空间, 不再是 平面的、缺乏参与性、仅供观赏的静态作品。除了智能 的穿戴设备, 还可以通过触摸屏与虚拟的电子系统进行 交互。交互式画册是基于具身认知理论进行设计的, 它 属于展示类的产品设计, 在画册中融入交互设计可以让 观者参与到绘画作品的再创造。人性化合理有趣的交互 设计可以提供给观者绝佳的浏览体验, 可以通过参与体 验, 了解画家的绘画技巧和绘制的过程, 可以体会画家 在创作作品过程的创作思维以及情感表达, 可以体会绘 画材料最终呈现的绘画效果, 从而更深刻的理解绘画作 品, 可辅助提升观者的艺术修养。

\section{4. 漆画图式体验的画册交互式设计}

漆画的图式在画册的交互设计中是以画布为基底 去呈现图式内容的, 画布便是笔触的感应区, 画布是基 于由像素点组成的位图处理方式的画布形式, 与画笔或 手指发生接触便会记录相应的像素点成像。画布的基底 可以通过交互改变原有漆画基底的色彩, 原有的漆画的 图式形式可以通过在交互菜单栏里改笔触的大小、粗 细、线条的样式、画笔的强度等进行尝试, 笔触的硬度 越高, 像素差值就越小, 图式骨骼越硬朗; 笔触硬度越 小, 差值越大, 会形成具有湿度的或厚重或缥纱的过渡 晕染的效果。这种模拟操作手法形式类似于 PHOTOSHOP 的界面菜单设计, 与其不同的是笔触的生成融入漆画常 用的肌理材料, 比如起皱的笔触, 铅丝、瓦灰、金银粉 等材料融入的笔触肌理。交互的手指操作可以模仿画笔 的操作, 用户可以对漆画作品进行临摹以及再创作, 对 于用户最终的呈现效果不做要求。目的在于让用户在临 摹的过程中去感受基于大漆材料的线条的笔触感和造 型能力。

\section{5. 漆画肌理体验的画册交互式设计}

著名漆画前辈乔十光先生在其《漆艺》一书中对漆 画常用的髹饰技法进行了介绍, 如描绘、镶嵌、变涂、 堆髹、磨显等。交互式画册的受众群体是对漆画感兴趣 的初学者, 是以培养对漆画的兴趣以及普及传播漆画画 种为目的, 更加注重应用自由的交互方式来吸引受众, 完成目标任务。【 ${ }^{31}$ 在实际的漆画创作中, 漆画创作者 具有较强的个人的主观创造性思维, 可以采用比较明确 的贴合自己创作想法的技法。对于受众而言，漆画的技 法更多的是让他们了解不同的技法在画面上的观感效 果。虽然数字漆画交互不能给与智能的量化的技法选 择，但是可以在同一种画面让受众尝试不同的技法呈现 的不同的绘画效果。

当线性的图式自主关系建立之后, 剩余的便是漆绘 肌理语言的表达。漆画的技法一部分来源于八千多年漆 艺的技法传承,一部分是基于艺术效果出发的创新型的 技法。在交互设计中, 要掌握漆画的技法的特点, 以“技 法样式板” 进行交互设计, 通过直观操作不同的表现技 法感受漆画技法的精髓。【4】要进行漆画技法的设计, 首要对各种漆画技法进行数据归类，彩绘、描金、研磨 髹涂、罩金、镶嵌、堆髹、雕漆等，在《髹饰录》中就 记载了上百种的漆艺技法, 加上在现代的漆画创作中, 漆画创作者自创的技法，这些技法的交互需要结合材料 的交互进行操作。漆画作品被识别成不同肌理的斑块模 式, 可点击进行局部斑块选择。

最终所要呈现的交互效果是: 用户选中其中一个斑 块肌理, 通过持续点击不同的按钮, 可以看到相应的画 面, 比如用户点击技法按钮, 可跳出常用的技法界面选 择, 选择想要尝试的技法点击, 可以看到选择的斑块画 面变成了选择的技法所呈现的肌理效果。比如原漆画中 运用描绘的技法, 如果替换成泼酒的技法, 最终会呈现 更加随机的、具有偶然性的肌理效果, 这样的方式一方 面可以加强用户兴趣体验，另一方面不同的技法也许可 以呈现全新的作品, 同时也适合漆画创作者的创作思路 的重建。在体验的过程中采用鼠标持续点击响应机制: 当菜单按钮被连续点击时, 变跳出图的显示属性, 鼠标 结束点击, 图片恢复到最初状态。交互设计的体验基于 最便捷、体验最优为原则。

\section{2 材料肌理的交互设计}

漆画的材料是漆画本身所具有的特性, 没有融入材 料的漆画作品是没有灵魂的, 能够入漆的材料很多, 如 蛋壳、螺钿、金银䈃、宝石、漆粉、瓦灰、麻布、豆类、 塑料袋、纸巾、羽毛等，一切可以对画面呈现效果有帮 助的材料都可以采用, 通过结合技法和材料所形成的肌 理具有不可重复性和不可预测性。不管是哪种材料, 都 会有漆独特的视觉艺术效果。不同的材料在形态、色彩、 质感上都存在差异，因此给与观者不同的视觉反应。

\section{1 技法肌理的交互设计}



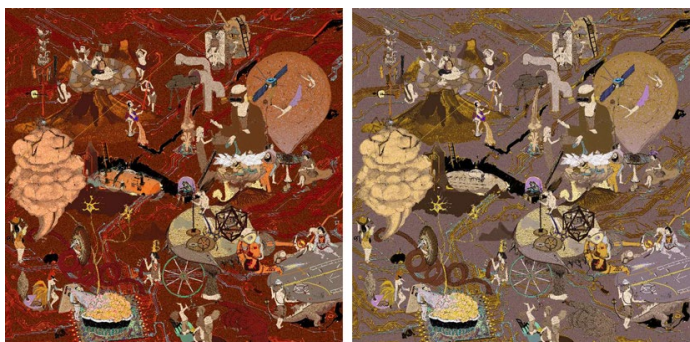

图 4 姚凯《中国芯-一生万物) 交互效果图 (图左为 未交互时的效果图，图右为交互后的效果图）

漆画材料肌理的交互设计仍然是基于不同的斑块 进行选择, 通过按钮操作, 用户可以查看菜单栏中不同 的材料, 同时也可看到不同的材料在漆画作品中呈现的 效果示意图。当用户点击蛋壳选项, 会有不同的蛋壳选 项, 如鸡蛋壳、鸭蛋壳、鸽子蛋壳, 同时会看到三种蛋 壳呈现的不同肌理示意图; 选择塑料袋选项, 下拉菜单 会有塑料袋的起皱程度, 调节强度横条可以直观的感受 塑料袋不同强度下随机的肌理效果; 选择豆类选项, 会 有两个下拉列表, 第一个是不同的豆子种类所形成的大 小尺度不同的豆坑，第二个是便是豆坑布局的几种随机 模式。选择完毕, 用户可以直接查看材料在图面上呈现 的效果, 也可以间接模仿创作者创作的顺序, 这样的交 互设计同样也特别适合漆画创作者在早期的草图阶段 创作稿的数字化绘制, 这种量化、便捷、直观的交互设 计可以同时创作出很多种绘画稿, 极大的提高了漆画创 作的效率; 对于用户来说，丰富的漆画材料直观的交互 体验也增加了用户的兴趣，激发用户使用产品的欲望， 可以给与感官带来舒适和愉悦, 沉浸感会更强。

\section{6. 交互式画册设计认知体验和可拓展性}

\section{1. 交互式画册设计的认知体验}

认知体验是整个交互式画册设计中核心目标, 用户 在具体的操作体验中可以学习并理解漆画图式、材料肌 理和技法肌理的精髓所在, 在赏析漆画作品时。可以运 用这些体验功能去更深层次的感悟漆画创作者的创作 思路, 另外可以开拓自己的艺术思维, 增加对漆画的兴 趣。对认知体验的测评重点在以下两个方面:

第一，用户能否准确理解漆画图式和肌理的特征。 学习和了解漆画的图式语言和肌理语言是整个交互画 册设计的指导思想, 在设计的导航菜单栏, 会对每个图 式、技法和肌理有名称、文字叙述、案例展示, 点击每 个选项, 菜单栏都会出现扩展的可以参与编辑创作的按 钮, 图式按钮在设计上要求简洁明了, 可以直观的通过 简化图标让用户了解其用途, 以期能适应各个年龄层次 用户人群。交互设计的最终评鉴需要对用户进行采访和 观察来调整。用户能否准备的理解漆画图式和肌理特征 仍需要更加深入的评测。

第二, 用户欣赏漆画时能够结合图式和肌理进行赏
析和再创作。赏析包含了两个层次的意义一欣赏和分 析。欣赏是直观的去看漆画作品, 通过观看画面引发共 鸣, 直面自己的内心感受。分析则是在欣赏的基础上, 去解读漆画作品的图式、肌理、创作者表达的用意。在 画册中的漆画作品都有高清大图可以观看画面的细节, 每幅作品有详细的分析, 可去查看较为权威的专家的解 读, 提升自身的赏析能力。交互式画册不仅提供给了用 户数字化的视觉情景体验, 并对用户的赏析做了延伸, 增加了很多互动参与创作的模块和功能, 帮助用户去深 入理解作品, “51掌握漆画这个画种的特点, 能简单了 对于作品进行微创作，提升用户的视觉敏感度。

\section{2. 交互式画册设计的可拓展性}

首先是交互方式的拓展。原有的交互形式从漆画的 图式和肌理两个方面做了参数设计, 模块功能具有较强 的漆画绘画特点。在以后的交互设计中，可以丰富模块 的功能, 可拓展成专业的漆画 APP 软件, 丰富其功能模 块, 比如增加强大的滤镜功能, 增加其专业性和精准程 度, 让初学者也可以完全描绘出漆画的韵味, 能够在临 摹的过程中感受漆画创作者的精妙的创作技能。

其次是交互用户的拓展。当交互设计做到功能丰富 强大和专业性，就无形中扩大了交互对象的使用面。通 常情况下, 漆画创作者的草图创作通常是手绘线稿、颜 料调色填充或借助制图软件比如 PHOTOSHOP 辅助创作, 呈现的草稿创作与最终的漆画作品往往有较大的差异 性, 究其原因就是漆画其图式和肌理的特殊性, 缺乏针 对漆画的专业的辅助工具。交互设计如果做到专业性, 会在草图阶段最大程度的 “还原” 漆画成品, 这会极大 的吸引漆画创作者的使用兴趣，开拓漆画创作者的思 路, 提升创作的数量和质量, 让漆画作品更多元化, 促 进漆画画种在未来向更优的方向发展。

\section{References}

[1] Zhang, Z., (2017) Research on ontology language of lacquer painting. Master thesis of nanjing university of the arts. 26-27.

Schema,360 encyclopedia, http://dict.youdao.com/search?q=encyclopedia\%0D\%0A\& keyfrom=fanyi.smartResult

[3] Yue.Y(2014) Research on the value of lacquer applied in modern lacquer art, Research on geomancy theory. Master thesis of jiangxi normal university of science and technology, Tianjin. 37-43.

[4] Guo, X, (2017), Research on interactive album design based on the differences of painting techniques. Master thesis of Harbin Institute of Technology,57-64.

[5] Chen X, Zhou MY (2019) User experience based interface design factor analysis, Design.,01: 81-83 